\title{
Stress levels of psychiatric nursing staff
}

\author{
P.C. de Looff ${ }^{1}$, E. Kuijpers ${ }^{2}$, H.L.I. Nijman ${ }^{1,3,4}$ \\ 1. Stichting De Borg, Den Dolder, The Netherlands. 2. GGZe, Eindhoven, The Netherlands. 3. Behavioral Science Institute, \\ Radboud University Nijmegen, The Netherlands. 4. Altrecht Aventurijn, Den Dolder, The Netherlands. \\ Correspondence: Henk Nijman. Address: Dolderseweg 164, Den Dolder, 3734 BN, The Netherlands. \\ Email: h.nijman@altrecht.nl
}

Received: November 18, 2013

Accepted: February 16, 2014

Online Published: April 1, 2014

DOI : $10.5430 /$ jnep.v4n7p1

URL: http://dx.doi.org/10.5430/jnep.v4n7p1

\section{Abstract}

During a total of 30 shifts, the arousal levels of 10 psychiatric nurses were assessed while working on a (forensic) psychiatric admissions ward. Arousal was assessed by means of a small device (wristband) by which the Skin Conductance Level (SCL) of the participating nurses was monitored. Each nurse was assessed during a full day, evening, and night shift. In the distribution of the SCL over the hours of the day, several elevations of the arousal levels were clearly visible during specific events. Additionally, an association was found between the neuroticism level of the staff members and their arousal level during their work.

\section{Key words}

Psychiatric nursing, Arousal, stress, Neuroticism, Workplace violence

\section{I ntroduction}

Results from a survey among 122 nurses indicated that psychiatric nursing as a specialty is considered the least respected in comparison to various other nursing specialties (e.g., oncology nursing, pediatrics, intensive care, labor and delivery nursing etc.; ${ }^{[1]}$ ). Psychiatric nurses were judged by the respondents ${ }^{[1]}$ to be less logical and dynamic, and seen as less skilled than nurses working in other domains of health care. Besides this negative image of the psychiatric nursing profession, mental health nurses, compared to the other nursing specialties, relatively often care for patients who lack insight into their own illness or condition. As a result of this, many psychiatric patients don't agree with their caregivers on the need to be hospitalized and treated to begin with. The fact that, psychiatric patients are often admitted against their will, makes building a good working alliance between the mental health professional and the patient relatively problematic in many respects. Considering this, it may not be surprising that nursing staff working on (locked) psychiatric admission wards, run a relatively high risk of exposure to situations in which patients may intimidate, harass and threaten them ${ }^{[2,3]}$. In addition, the workload of psychiatric nurses is increasing ${ }^{[4]}$, and psychiatric nurses generally are expected to work in alternating day, evening and night shifts. All of these challenging characteristics (e.g., the negative image of the mental health profession, the exposure to aggressive and threatening behaviors, the high workload) of the mental health nursing profession may make the job relatively exhausting and stressful compared to other professions.

Findings from a survey study indicated that exposure of psychiatric staff members to aggression from their patients is also directly associated with an increased risk of having to call in sick for work ${ }^{[3]}$. As is the case with many other health care 
professions, many psychiatric nurses quit the profession each year. According to Happell ${ }^{[4]}$ a major cause of this is: “... because they are dissatisfied with the working conditions and find the associated physical and mental distress to be overwhelming". Apart from research on the prevention of workplace violence ${ }^{[5]}$, and trying to create "a positive workplace culture" ${ }^{[4]}$ to retain nurses in the profession, it may well be that certain personality characteristics are needed to be able to deal with the many stressful, complex and unexpected situations that a psychiatric nurse encounters in his or her career. In a study among Dutch psychiatric emergency staff, it was found that caregivers working at a psychiatric emergency response unit had lower neuroticism levels in comparison to the general population ${ }^{[6]}$. Individuals with strong neurotic traits have: “(...) the tendency to experience negative, distressing emotions and to possess associated behavioral and cognitive traits. Among the traits that define this dimension are fearfulness, irritability, low self-esteem, social anxiety, poor inhibition of impulses, and helplessness.” ${ }^{[7]}$. In the United Kingdom, Bowers et al. ${ }^{[8]}$ investigated the characteristics of staff members involved with the care of so-called "DSPD-patients” (Dangerous and Severe Personality Disorders) and found a negative correlation between Neuroticism and how secure these staff members felt in working with these DSPD-patients.

In the current small-scale pilot study, the arousal levels of a group of psychiatric nurses were assessed during their work on a (forensic) psychiatric admissions ward. The arousal level in this respect was operationalised in terms of the Skin Conductance Levels (SCLs) of the nurses, which rise as the activity of sweat glands increases, as a concomitant of physiological, psychological and emotional states which appear under stress ${ }^{[9]}$ (for more information also see the "Methods" section of this paper). The aim of this study was to investigate whether specific working times were associated with higher levels of arousal and to gain insight into which ward activities were related to increased arousal levels. In addition, the association between certain personality traits of staff members and their arousal levels during their work were investigated. It was hypothesized that nurses with strong neurotic traits in particular would also show higher levels of arousal during their work on the ward.

\section{Methods}

\subsection{Study design}

It concerns an exploratory pilot study conducted on a locked (forensic) psychiatric admissions ward in the middle of the Netherlands. The ward houses two separate units that share a joint staff office. These two units contain eleven and ten beds, respectively. These 21 admission beds are designated for the treatment of newly admitted (forensic) psychiatric patients of whom the vast majority suffer from florid psychotic symptoms.

Ten psychiatric nurses volunteered to wear small devices in the form of wristbands, by which their SCLs were recorded continuously, during a total of 30 shifts. To be more specific, each of the 10 participating staff members wore the device during a morning, evening and a night shift. This piloting of these devices on the $10 \mathrm{staff}$ members was done to prepare for a larger scale study on the associations between (rises of) SCLs of psychiatric patients and aggressive incidents. In figure 1 , one of the used devices, which are called “Q-sensors”, is depicted.

Figure 1. Photograph of a Q-sensor used to access the skin conductance levels of staff members during a day, evening, and night shift

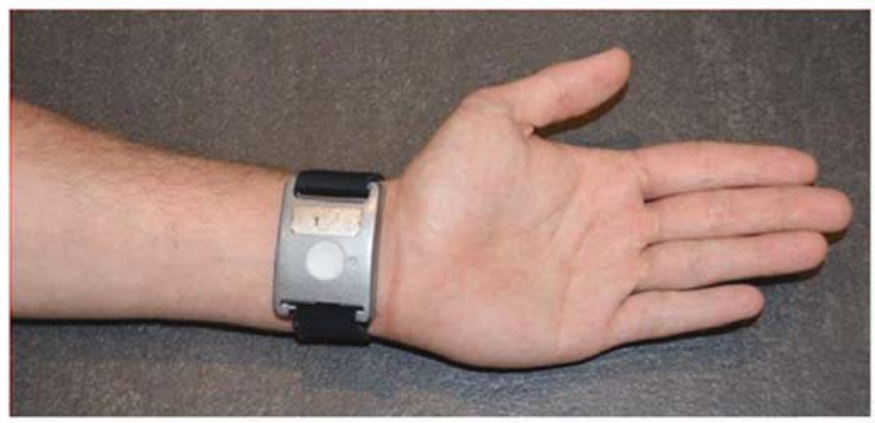


To assess the neuroticism of the participating nurses, they also completed the Neuroticism, Extraversion and Openness Five factor Inventory (i.e., the NEO -FFI in brief) developed by Costa \& McCrea ${ }^{[10]}$. Underneath both assessments (i.e., the assessment of arousal in terms of the SCLs and the assessment of neuroticism by means of NEO-FFI) are addressed in more detail.

\subsection{Assessments}

\subsubsection{Assessment of the arousal levels}

Measurements of Skin Conductance Levels (SCLs), and rapid changes in skin conductance in response to triggers (Skin Conductance Responses; SCRs), have been used to assess (psychological) arousal for well over a century now ${ }^{[9]}$. SCLs and SCRs are assessed on the basis of the electrodermal resistance of the skin. The electrodermal resistance of the skin reduces and the conductance increases as the activity in the sweat glands increases. Changes in sweat gland activity in response to stressful or threatening stimuli occur very rapidly (in a few seconds skin conductance levels can increase drastically) and are not under voluntary control of subjects. In experimental psychological research in particular, for instance in samples suffering from phobia ${ }^{[11]}$, this technique has been often used. The number of studies of SCLs of psychiatric patients while being admitted on (locked) psychiatric admission settings, however, is limited ${ }^{[12]}$. One of the obvious reasons for this is that measuring skin conductance, until recently, required a substantial amount of equipment to which the participant had to be attached to obtain these assessments. As far as we know, the studies that have been conducted on locked psychiatric admissions wards so far have all focussed on the arousal levels of the patients, but not on those of staff members who have to take care, and manage, the sometimes disruptive and aggressive behaviour of their patients.

In the current study, the SCLs of the participating nurses were recorded at two Hertz during a full day, evening, and night shift by means of a "Q-sensor" (see Figure 1). In other words, for each of the staff members a full assessment of their arousal level while being on duty was obtained 'around the clock'.

\subsubsection{Assessment of personality traits}

The personality characteristics of the 10 participating nurses were assessed by means of the NEO-FFI ${ }^{[10]}$. The NEO-FFI is a brief version of the Neuroticism, Extraversion and Openness - Personality Inventory - Revised (i.e., the NEO -PI-R in brief) and was developed by Costa \& McCrae ${ }^{[10]}$. The NEO-FFI is a self-report instrument consisting of 60 items by which five main personality characteristics are assessed. These five personality traits, which are also referred to as the Big Five, are: Openness, Conscientiousness, Extraversion, Agreeableness, and Neuroticism. In the current study, it was hypothesized that Neuroticism in particular would be positively associated with a higher mean SCL of the psychiatric nurses while being on duty. The reliability and validity of the NEO-FFI have been found to be satisfactory to good in a number of studies ${ }^{[10,13]}$ and for the Neuroticism scale in particular. To illustrate this, in the study of Holden ${ }^{[13]}$, the reliability (internal consistency) of the Neuroticism scale was 0.87 , and the convergent validity with the "Psychiatric Symptomatology" and the "Depression” scales from the Holden Psychological Screening Inventory were 0.61 and 0.63, respectively ${ }^{[13]}$.

\subsection{Statistical analyses}

As the SCLs were not normally distributed (i.e. with the skewness of the distribution of electrodermal activity being 1.9 and a kurtosis of 4.1), a Friedman nonparametric test and a Wilcoxon rank sign test were used instead of an ANOVA. It was investigated which shift (day, evening or night shift) on average seems to be most stressful in terms of elevated SCLs. The association between the mean SCLs of the 10 nurses and their personality characteristics were explored by means of Spearman's r correlations. In these analyses, it was hypothesized that Neuroticism in particular would be connected to elevated (mean) SCL scores ${ }^{[14]}$ (but it should be noted that results on this in a general sense are inconsistent; see Boucsein ${ }^{[9]}$ ). The association between neuroticism as measured with the NEO-FFI and the mean SCL for this reason was tested one-tailed with an alpha set at 0.05 . The associations between the other four NEO-FFI personality dimensions (i.e., 
Openness, Conscientiousness, Extraversion and Agreeableness) and the mean SCL, were explored by two-tailed tests, with alpha set at 0.05 . Finally, an anecdotal description of a situation is provided in which the arousal level of an individual staff member 'spiked' during a shift. This is done to provide anecdotal 'evidence' of the impact certain typical tasks that psychiatric nurses have to perform, can have on their stress levels.

\section{Results}

\subsection{Sample characteristics}

The sample of 10 staff members consisted of seven women (70\%) and three men (30\%). Their average age was 30.2 years (s.d. = 7.7; range $23-46$ years). Their scores on the big five personality traits as assessed with the NEO-FFI were 29.0 (s.d. $=5.5 ; 4.4^{\text {th }}$ decile on average) for Neuroticism, 42.8 (s.d. $=5.1 ; 5.8^{\text {th }}$ decile on average for Extraversion, 39.5 (s.d. = 3.8; 6.2th decile on average) for Openness, 46.1 (s.d. $4.0 ; 5.7^{\text {th }}$ decile on average) for Altruism, and 46.3 (s.d. $=5.4 ; 5.5^{\text {th }}$ decile on average) for Conscientiousness.

\subsection{SCLs during day, evening and night shifts}

In figure 2, the mean SCL of the 10 participating psychiatric nurses is depicted per 15 minutes. A look at this figure suggests that the SCL levels are highest during evening shifts. A Friedman non parametric test (as the SCL scores are not normally distributed) indicates that the mean SCL indeed significantly differs during the three shifts [Chi-square (2) = 7.8; $p=.02]$.

In order to test the contrasts between the separate shifts, a Wilcoxon signed ranks test was used. The results indicate that the mean SCL of the nurses during evening shifts was elevated compared to both the day $(p=.038)$, as well as to the night shift $(p=.012)$. The difference between the day and the night shift did not reach significance $(p=.49)$. In terms of the mean SCL of the 10 nurses, the mean SCL during evening shifts was $3.2 \mu$ Siemens (s.d. $=2.6$ ), during night shifts the mean SCL was $1.8 \mu$ Siemens (s.d. $=1.7$ ), and during day shifts it was $1.7 \mu$ Siemens (s.d. $=1.0$ ) on average.

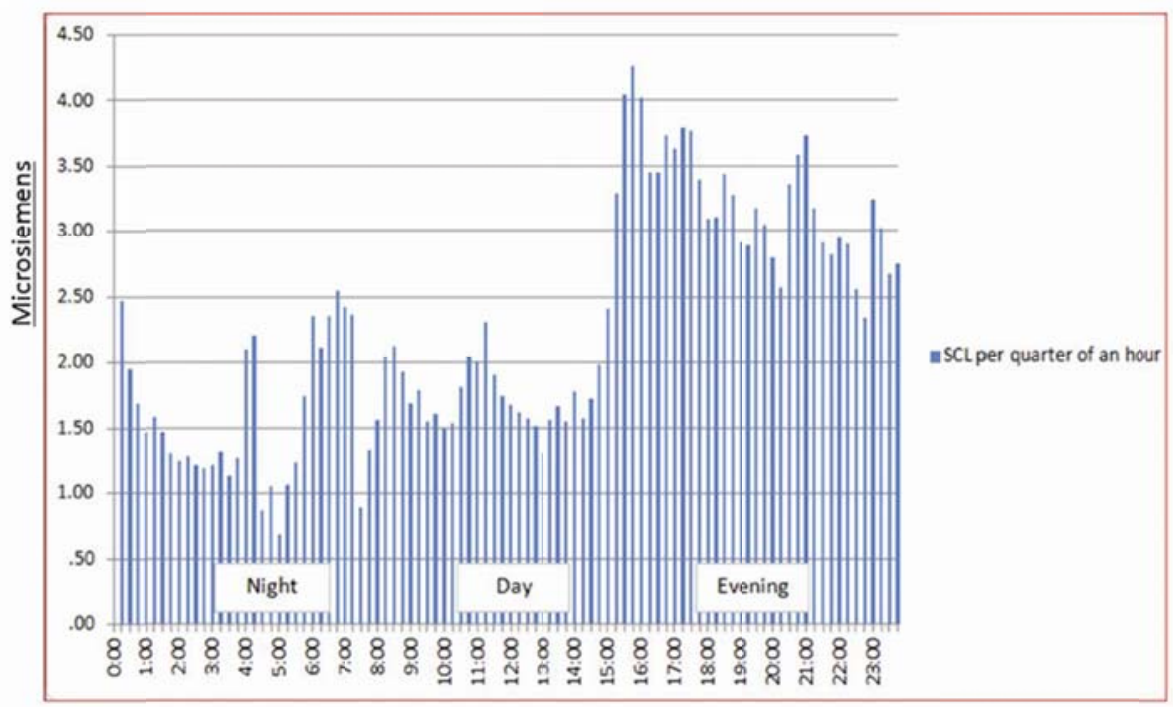

Figure 2. The SCL of the 10 participating psychiatric nurses depicted per quarter of an Hour

Apart from the overall increase during the evening shift, several relative increases of the SCL are visible at specific time frames in the graph. Visual inspection of the graph, going from left to right, reveals a first relative 'spike' of the average SCL between 4 and half past 4 during the night. Inquiry with the nursing staff revealed that each night a test of the alarm 
system of the ward is performed in that time frame. The noise of the alarm, which is likely to have been conditioned with real stressful situations on earlier occasions, possibly arouses a strong relative increase of the SCR. The next and more prolonged elevation of the SCL can be seen between 5.30 and 7 AM. The nurses we asked about this, attributed this elevation to having to prepare for the next shift, which means they have to inspect the ward, prepare the handover documentation, while at the same time, as the nurses of the ward put it, they are: "often are struggling hard to stay awake" in that time frame. The next small spike is visible around 8 to 9 AM. During this time patients (have to) get up, breakfast has to be prepared and many patients have to take their first medication. Again some modest elevation of the SCL between 11 and 12 AM, possibly being connected to handing out a second dosage of medication and preparing for lunch.

The most pronounced 'spike' of the SCL is between 3 and 4 PM, at 3 PM the evening shift comes in and a hand-over meeting is conducted. After that, the new shift goes to work on the ward. At around 5 PM, a modest relative elevation again is visible, which may be connected to the group gathering with all patients which takes place each day to evaluate the day, and the (preparation of) the evening meal following that meeting. Around 9 PM another relative increase of the SCL is visible. According to the ward nurses, this elevation may be connected to the ward rule that the patients are allowed at this time to get some more food and drinks for themselves from the kitchen. At that time, almost all patients leave their rooms and gather in the kitchen and living room. At $10 \mathrm{PM}$ another small elevation is seen. At this time patients are expected to retreat to their rooms for the night. Finally a relative elevation of the SCL is visible at around 11 PM which may be connected to a walking round at that time in which the ward and patients rooms are inspected. According to the nurses, arguments over (sleeping) medication are also relatively common at this time of the day.

\subsection{Associations between personality characteristics and the SCL}

In Table 1 the (Spearman's rho) correlations between the personality traits as assessed with the NEO-FFI norm scores and the mean SCL of the 10 psychiatric nurses are presented. As can be seen in this table, Neuroticism is most strongly and positively associated with the arousal level of the nurses (Spearman's rho $=0.58 ; p<.05$; one-tailed). In other words, the results indicate that the higher a psychiatric nurse scored on Neuroticism, the higher the (psychological) arousal was while being on duty.

Table 1. Spearman's rho correlations between the NEO-FFI personality norms scores and the mean SCL of the 10 psychiatric nurses

\begin{tabular}{llllll}
\hline & Neuroticism & Extraversion & Openness & Altruism & Conscientiousness \\
\hline \multirow{3}{*}{ Mean SCL } & $\boldsymbol{r}=\mathbf{0 . 5 8}$ & $\boldsymbol{r}=\mathbf{0 . 3 7}$ & $\boldsymbol{r}=\mathbf{- 0 . 3 5}$ & $\boldsymbol{P}=\mathbf{0 . 3 7}$ & $\boldsymbol{P}=\mathbf{- 0 . 4 3}$ \\
& $p=.04$ & $p=.29$ & $p=.33$ & $p=.30$ & $P=.22$ \\
& (one-tailed) & (two-tailed) & (two-tailed) & (two-tailed) & (two-tailed) \\
\hline
\end{tabular}

\subsection{An illustration of a stress inducing nursing task}

To illustrate how some of the tasks psychiatric nurses have to perform can impact their arousal level, the SCL level of nurse X. is depicted in Figure 2. This nurse was informed that it was decided that Mr. A, a male psychotic patient who had displayed verbally threatening and intimidating behaviour for days in a row, would be required to take medication. In Figure 2, the SCL of Nurse X. is displayed at the moment he was informed that he would have to be the so-called "spokesperson" about this issue to Mr. A.. In other words, nurse X. realises he has to bring this news to this severely agitated patient of which the psychiatrist and nursing team believe that he is already at the verge of engaging in assaultive behaviour. On this ward, it is policy to appoint one staff member as a spokesperson in case of difficult news. The moment nurse X. is being informed that he is appointed to explain to the patient he is expected to take medication, his SCL briefly spikes (see Figure 3), but his SCL also ‘recovers’ very quickly, returning near to the level before the spike. 


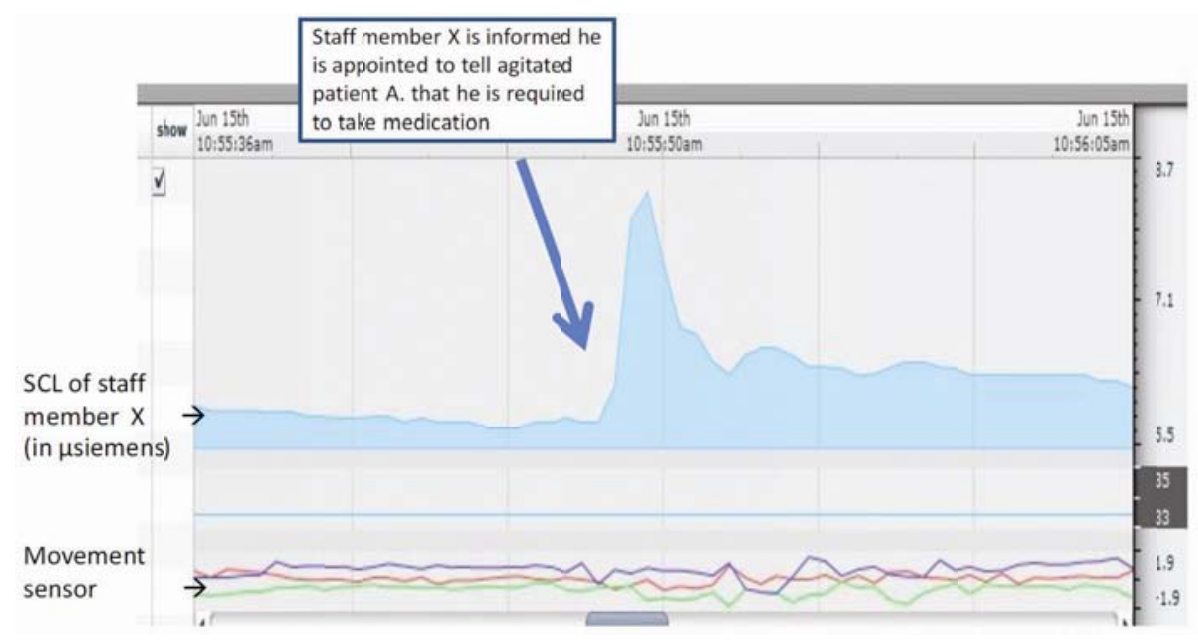

Figure 3. Illustration of the influence of a stress inducing nursing task on the SCL

\section{Discussion}

Even though the current study was a small scale pilot study, some significant associations with the arousal levels of the studied nurses were found. To begin with, the mean SCL of the ward nurses was higher on average during the evening shift, compared with the day and night shifts. Even though there are indications that SCLs on average vary during the hours of the day ${ }^{[15,16]}$, the found differences in the current study were marked, with the evening hours consistently showing higher (mean) SCLs compared to working hours during the day and night shifts. During the evening shift, the number of nurses on duty is the same as during the day shift, whereas the number of potentially stressful tasks (e.g., preparing and supervising evening diner, requiring patients to go to their room and stay there for the night) and situations (e.g., arguments about (sleeping) medications), as well as the number of patients on the ward, is high. While during the day, some of the patients may be gone to therapies or other activities outside the ward, all patients usually are locked in together on the ward during the evening shift. The present pilot study suggests that the arousal level of nurses during the evening shift is almost twofold that of the dayshift (i.e., mean SCLs being $3.2 \mu$ Siemens during evening shifts versus $1.7 \mu$ Siemens during day shifts), whereas the staffing level during the evening is equal to that during the day shift, while at the same time there are more patients at the ward during the evening on average.

Furthermore, in the current limited sample we found indications that the mean SCL while working on the ward was associated with the level of neuroticism of the nurses. Although highly speculative, it possibly is the capacity to recover from stressful events, in terms of a rather quick return of the arousal level near to baseline what makes a person being able to perform well and stay in the psychiatric nursing profession over a prolonged time. With the constant potential for unexpected incidents and situations to occur during a shift, the risk of an elevated arousal level not getting the chance or time to return to baseline levels may exist, which may eventually lead to exhaustion from a more chronic state of stress during working hours. Indeed, Norris, Larsen \& Cacioppo ${ }^{[14]}$ demonstrated that persons with high neuroticism scores not only show more pronounced SCRs, but also have somewhat longer 'recovery' times to return to the SCL before the SCR. The psychiatric nursing job in particular, especially when working on a locked admissions ward, may be a job that has so many minor and major events happening all the time, and offers so little 'predictability', that staff members with strong neuroticism traits in particular, may not get enough time to recover from incidents or cognitions that impact the arousal level, leading to a state in which discrete SCRs sort of start 'overlapping,' or even 'stacking on' to, each other, which can result in a constant state of high arousal while on duty. In a general sense, real breaks from the job, such as coffee or lunch breaks, could possibly prevent this, but in practice, however, coffee breaks are often enjoyed at the staff office, or somewhere on the ward, which means that the nurse will still be noticing what is happening on the ward, and perhaps still be answering the ward phone and taking questions from patients. In case of an incident, the nurse on break is also expected 
to respond. Circumstances like these are likely to prevent breaks from having a substantially decreasing effect on the SCL. Clearly, speculations like these need further research in a much larger sample of psychiatric nurses and to be tested with a longitudinal design in which the mean SCL level of the participants can be connected to (future) rates of sick leave and attrition from the psychiatric nursing profession. In a study like that the reasons for leaving the profession should also be assessed in detail. With the very limited sample size of the present study in mind, the suggestions made above are only hypothetical and not more than that. Considering the high rates of sick leaves and attrition from the psychiatric nursing profession, however, we think such a study is warranted, and feel that the assessment of neuroticism along with the arousal levels of psychiatric nurses during their work, possibly even in direct connection to, and interaction with, the arousal levels of the patients they take care of, could be valuable to gain more insight in what makes the psychiatric nursing profession into such a difficult job.

\section{Competing interest}

The Q-sensors were obtained from Affectiva, see: http://www.qsensortech.com/. The authors of this paper have no commercial or other ties with this company

\section{References}

[1] Halter, M.J. Perceived characteristics of psychiatric nurses: Stigma by association. Archives of psychiatric nursing. 2008; $22: 20-26$. PMid:18207053 http://dx.doi.org/10.1016/j.apnu.2007.03.003

[2] Nijman, H.L.I., Allertz, W.W.F., Merckelbach, H.L.G.J., à Campo, J.M.L.G. Ravelli D.P. Aggressive behaviour on an acute psychiatric admissions ward. European Journal of Psychiatry. 1997; 11: 106-114.

[3] Nijman, H.L.I., Bowers, L., Oud, N. \& Jansen, G. Psychiatric nurses’ experiences with inpatient aggression. Aggressive Behavior. 2005; 31: 217-227. http://dx.doi.org/10.1002/ab.20038

[4] Happell, B. Retaining our nurses: Why aren’t we ahead of the pack? International Journal of Mental health Nursing. 2009 ; 18 : 1. PMid:19125780 http://dx.doi.org/10.1111/j.1447-0349.2008.00589.x

[5] Van de Sande, R., Nijman, H., Noorthoorn, E., Wierdsma, A., Hellendoorn, E., van der Staak, C. \& Mulder, N. Reduction of aggression and seclusion by short-term risk assessment on acute psychiatric wards; a cluster randomized trial. British Journal of Psychiatry. 2011; 199: 473 -478. PMid:22016437 http://dx.doi.org/10.1192/bjp.bp.111.095141

[6] Penterman, B., Smeets, J., van der Staak, C, Özer, H. \& Nijman, H. Persoonlijkheidskenmerken van crisisdienst medewerkers in de GGZ. Tijdschrift voor psychiatrie. 2011; 53: 145-151. PMid:21404171

[7] Costa, P. T. \& McCrae, R.R. Neuroticism, somatic complaints, and disease: is the bark worse than the bite? Journal of personality. 1987; 55: 299-316. PMid:3612472 http://dx.doi.org/10.1111/j.1467-6494.1987.tb00438.x

[8] Bowers., L., Callaghan, P., Nijman, H., Paton, J., Carr-Walker, P., Allan, T. et al. The Right People for the Job: choosing staff that will adjust positively and productively to working in the new personality disorder (PD) services (report to the home office). London: City University, 2003.

[9] Boucsein, W. Electrodermal activity (second edition). New York: Springer, 2012. PMCid:PMC3343236 http://dx.doi.org/10.1007/978-1-4614-1126-0

[10] Costa, P.T., McCrae, R.R. Revised NEO Personality Inventory (NEO-PI-R) and NEO Five-Factor Inventory (NEO-FFI) manual. Odessa, FL: Psychological Assessment Resources, 1992.

[11] De Jong, P.J., Merckelbach, H.L.G.J., Arntz, A., Nijman, H.L.I. Covariation Bias in treated and untreated phobics. Journal of Abnormal Psychology. 1992; 101: 724-727. PMid:1430613 http://dx.doi.org/10.1037/0021-843X.101.4.724

[12] Campo, J.M.L.G., Merckelbach, H.L.G.J., Nijman, H.L.I., Allertz, W.W.F. Yeates-Frederikx, M. Schizofrene symptomatologie en huidgeleidingsactiviteit [Schizophrenic symptomatology and skin conductance activity]. Acta Neuropsychiatrica. 2000; 12: 177-182.

[13] Holden, R. R. Associations between the Holden Psychological Screening Inventory and the NEO Five Factor Inventory in a non-clinical sample. Psychological Reports. 1992; 71: 1039-1042. http://dx.doi.org/10.2466/pr0.1992.71.3f.1039

[14] Norris, C. J., Larsen, J. T., \& Cacioppo, J. T. Neuroticism is associated with larger and more prolonged electrodermal responses to emotionally evocative pictures. Psychophysiology. 2007; 44: 823-826. PMid:17596178 http://dx.doi.org/10.1111/j.1469-8986.2007.00551.x

[15] Hot, P., Naveteur, J., Leconte, P., \& Sequeira, H. Diurnal variations of tonic electrodermal activity. International Journal of Psychophysiology. 1999; 33: 223-230. http://dx.doi.org/10.1016/S0167-8760(99)00060-4

[16] Hot, P., Leconte, P., \& Sequeira, H. Diurnal autonomic variations and emotional reactivity. Biological Psychology. 2005; 69: 261-270. PMid:15925029 http://dx.doi.org/10.1016/j.biopsycho.2004.08.005 